\title{
A Structured, Manual-Based Low-Level Intervention vs. Treatment as Usual Evaluated in a Randomized Controlled Trial for Adolescents with Extreme Obesity - the STEREO Trial
}

\author{
Yvonne Mühlig $^{\mathrm{a}}$ André Scherag $^{\mathrm{b}} \quad$ Annika Bickenbach $^{\mathrm{c}} \quad$ Ulrike Giesen $^{\mathrm{a}}$ \\ Reinhard Holl ${ }^{d}$ Rolf Holle ${ }^{e}$ Wieland Kiess ${ }^{f}$ Belinda Lennerz ${ }^{g}$ \\ Diana Lütke Brintrup ${ }^{h}$ Anja Moss ${ }^{g}$ Madlen Neef $^{f}$ Claudia Ose $^{h}$ \\ Thomas Reinehr $^{i} \quad$ Christina M. Teuner ${ }^{\mathrm{e}}$ Susanna Wiegand ${ }^{\mathrm{C}}$ \\ Barbara Wolters $^{i}$ Martin Wabitsch ${ }^{9}$ Johannes Hebebrand $^{\text {h }}$

\begin{abstract}
a Department of Child and Adolescent Psychiatry, Psychosomatics and Psychotherapy, University Hospital Essen, University of Duisburg-Essen, Essen, Germany; ${ }^{b}$ Research Group Clinical Epidemiology, Integrated Research and Treatment Center, Center for Sepsis Control and Care (CSCC), Jena University Hospital, Jena, Germany; ${ }^{\mathrm{C}}$ Ambulatory Obesity Center, Charité - University Hospital Berlin, Berlin, Germany; ${ }^{d}$ Institute for Epidemiology and Medical Biometry, Ulm University, Ulm, Germany; ${ }^{e}$ Institute of Health Economics and Health Care Management, Helmholtz Zentrum München - German Research Center for Environmental Health, Munich, Germany; ${ }^{f}$ Hospital for Children and Adolescents, Department of Women and Child Health, University of Leipzig, Leipzig, Germany; ${ }^{9}$ Division of Pediatric Endocrinology and Diabetes, Interdisciplinary Obesity Unit, Department

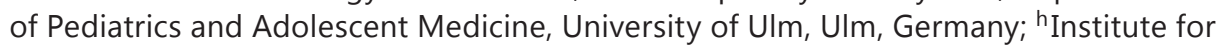
Medical Informatics, Biometry and Epidemiology and Center for Clinical Trials Essen (ZKSE), University Hospital Essen, University of Duisburg-Essen, Essen, Germany; ${ }^{\text {VVestische }}$ Childrens Hospital Datteln, University Witten/Herdecke, Datteln, Germany
\end{abstract}

\section{Keywords}

Extreme obesity · Youth · Adolescents · Adherence $\cdot$ Quality of life $\cdot$ HRQoL

\section{Abstract}

Background: To compare efficacy and safety of a manual-based low-level psychological intervention with treatment as usual (weight loss treatment). Methods: A two-armed randomized controlled trial without blinding and computer-based stratified block randomization included adolescents and young adults (14.0-24.9 years) with a BMI $\geq 30 \mathrm{~kg} / \mathrm{m}^{2}$ at five German university hospitals. Primary outcomes were adherence (participation rate $\geq 5 / 6$ sessions) and quality of life (DISABKIDS-37) 6 months after randomization. Secondary outcomes included

Dr. rer. medic. Yvonne Mühlig

Department of Child and Adolescent Psychiatry, Psychosomatics and Psychotherapy LVR-Klinikum Essen, University of Duisburg-Essen

Wickenburgstraße 21, 45147 Essen, Germany

yvonne.muehlig@Ivr.de 
Mühlig et al:: A Structured, Manual-Based Low-Level Intervention vs. Treatment as Usual Evaluated in a Randomized Controlled Trial for Adolescents with Extreme Obesity - the STEREO Trial

depression, self-esteem, and perceived stress scores. Results: Of 397 screened adolescents, 119 (mean BMI $40.4 \pm 7.0 \mathrm{~kg} / \mathrm{m}^{2}, 49.6 \%$ female) were randomized to the manual-based lowlevel intervention $(n=59)$ or treatment as usual $(n=60)$. We observed no group difference for adherence (absolute risk reduction $0.4 \%, 95 \% \mathrm{CI}-14.7 \%$ to $15.5 \%$; $\mathrm{p}=1.0$ ) or health-related quality of life (score difference $8.1,95 \% \mathrm{CI}-2.1$ to $18.3 ; \mathrm{p}=0.11$ ). Among all secondary outcomes, we detected explorative evidence for an effect on the DISABKIDS-37 'social exclusion' subscale (score difference 15.5; 95\% CI 1.6-29.4; $p=0.03$ ). 18/19 adverse events occurred in 26 participants, none were classified as serious. Conclusion: Adherence to a coping-oriented intervention was comparable to weight loss treatment, although it was weak in both interventions. Psychological interventions may help to overcome social isolation; further confirmation is required.

(C) 2017 The Author(s)

Published by S. Karger GmbH, Freiburg

\section{Introduction}

Worldwide, $5 \%$ of children and adolescents are affected by obesity [1]. Weight loss programs for children and adolescents lead to statistically significant effects on weight status, but the overall amount of weight reduction is modest [2,3]. One major problem in evaluating the effects of such treatments is the high attrition rate of up to $42-50 \%[2,3]$. Determinants of the frequent 'drop out' are poorly understood.

Based on data from systematic reviews [2,3] and clinical trials [4], adolescents with extreme obesity (BMI $\geq 99.5$ th percentile) cannot be expected to reach substantial weight loss through lifestyle interventions over the intermediate term. Extreme obesity entails a high risk of somatic [5] and psychiatric [6] co-morbidities. Extremely obese adolescents face stigmatization in peer groups [7] and work settings, entailing the risk of long-term unemployment $[8,9]$. Accordingly, an increasing number of adolescents are treated with bariatric surgery [10]. Weight loss surgery appears to be the only effective treatment for extreme obesity regarding weight loss and improvement of co-morbidities $[11,12]$.

While conservative treatment may lead to feelings of failure regarding weight reduction, coping with obesity-related psychosocial impairments is not regularly part of the standard care. Effects of obesity treatment on psychosocial outcomes have infrequently been evaluated in previous trials. In our review [3], only one of the included randomized controlled trials (RCTs) defined self-efficacy as a primary outcome [13], even though the focus of the intervention was on weight loss. We are not aware of any RCT with a focus on adherence as the primary outcome in an intervention, in which weight loss was not a treatment goal.

Adherence has frequently been measured by the participation rate in an intervention [3, 14]. Additionally, the term adherence has been applied to behavioral targets (e.g. healthy diet/activity behaviors) $[15,16]$. In light of the overall weak effects of weight loss treatment on weight status in extremely obese adolescents [2-4], we concentrated on the participation rate as the main and sole measure of adherence, which is our primary outcome. The participation rate in an intervention aimed at promoting coping strategies and acceptance of obesity represents an appropriate type of outcome for this extreme weight group. We assume that a successful participation may represent an important indicator of long-term adherence of patients who subsequently receive bariatric surgery. Long-term adherence may in turn help minimizing possible complications after bariatric surgery due to continuous monitoring. Provided that this assumption is correct, assessment of adherence may also help to identify individuals eligible for bariatric surgery.

Acknowledging the limited efficacy of weight loss treatment and potentially related feelings of failure and shame, we hypothesized that adolescents and young adults with 
Mühlig et al.: A Structured, Manual-Based Low-Level Intervention vs. Treatment as Usual Evaluated in a Randomized Controlled Trial for Adolescents with Extreme Obesity - the STEREO Trial

extreme obesity may show a greater adherence in a treatment program with the aim of coping with obesity rather than weight loss. Thus, this RCT was designed to evaluate i) efficacy and ii) safety of a manual-based low-level intervention aimed at improving quality of life, mood, self-esteem, and perceived stress compared to treatment as usual. We hypothesized that i) adherence to the program and ii) covariate-adjusted changes in quality of life between baseline and the 6-month follow-up differ between the two treatment groups.

\section{Material and Methods}

\section{Trial Design}

The STEREO trial was designed as an open two-armed RCT conducted at five centers in Germany (Pediatric University Hospitals in Ulm, Datteln, Berlin and Leipzig, and Child and Adolescent Psychiatry in Essen) that randomized 13/32/38/0/36 participants. The STEREO trial was part of the Youth with Extreme Obesity Study (YES) [17].

\section{Participants}

Participants were recruited between September 2012 and June 2014. The 6-month follow-up assessments were conducted from March 2013 to December 2014. Recruitment pathways involved regional outpatient obesity care units and advertisement via internet, media, and job centers [17]. In Essen, recruitment was mainly based on a co-operation with the job center with a focus on unemployed young subjects aged between 15.0 and 24.9 years, and the diagnostic and therapeutic procedures were offered on the premises of this job center. An age range from 14.0 to 24.9 years (42/119 randomized participants were aged $\geq 18$ years) and a BMI $\geq 30$ $\mathrm{kg} / \mathrm{m}^{2}$ formed the inclusion criteria. In March 2013, the former inclusion criterion of an age range 14.0-20.9 years was modified by an amendment of the study protocol in order to also reach the high-risk group of young adults and to stimulate recruitment. Moreover, the traveling time spent between residence and center had to be below 90 min using either private or public transportation. Exclusion criteria were insufficient knowledge of German language, intellectual disability, and conditions precluding an outpatient treatment (i.e. immediate or imminent requirement of hospitalization due to severe somatic or psychiatric disorders).

The collected baseline characteristics included sex, age, native country, measured height and weight for calculation of BMI, and self- or participant-reported parental BMI, parental educational status, and parental nationality.

\section{Interventions}

The STEREO trial had two intervention arms [17]: an innovative manual-based low-level intervention focusing on coping with obesity and promoting quality of life and an interdisciplinary lifestyle intervention aimed at weight reduction (treatment as usual (TAU)). Both interventions were performed on the basis of a structured manual to ensure consistency between study centers. Each program consisted of six 90-min group sessions offered every 2 weeks. Thus, the intervention was classified as low-level. The same amount of sessions for each program allowed for comparison. Prior to the randomization, participants were informed that based on the current scientific evidence no superiority of one of the interventions concerning weight loss is assumed. Participants of both interventions who attended at least five sessions were offered participation in a subsequent information and preparation program on bariatric surgery ([17]; data not presented); this information was provided at baseline.

\section{Manual-Based Low-Level Intervention}

The overarching aim of this intervention was coping with obesity and its acceptance. Importantly, weight reduction was not a focus. The manual-based low-level intervention program included elements of cognitive behavior therapy and motivational interviewing. It was delivered by trained psychologists and 
Mühlig et al.: A Structured, Manual-Based Low-Level Intervention vs. Treatment as Usual Evaluated in a Randomized Controlled Trial for Adolescents with Extreme Obesity - the STEREO Trial

involved the topics life satisfaction (e.g. family, friends, and school/work), self-esteem (e.g. concentrating on other strengths than physical appearance), social competency (e.g. initiating friendships), body image (e.g. acceptance of body shape), and coping with bullying and anger. The sessions comprised psychoeducative elements, role-playing, body image exposition or response prevention (avoidance of looking at or excessively checking one's own body shape), activation, problem solving, and therapeutic homework to consolidate the learned skills. During and after the intervention, the participants had access to a local expert network (e.g. psychotherapist, vocational counseling) depending on their own needs.

TAU

The overarching focus of TAU was weight reduction. The TAU program corresponded to the recommendations of the German evidence-based guidelines for the treatment of obesity in childhood and adolescence [18]. It was delivered by a multiprofessional team involving trained psychologists, physicians, dieticians, and physical activity therapists. The topics were medical information (causes, consequences, and treatment options of obesity), nutrition (healthy food choices and frequency of meals), eating behavior (e.g. distinguishing between hunger and appetite, learning action alternatives for emotional eating), self-esteem (focusing on personal strengths), and media consumption (reduction of sedentary behavior).

\section{Outcomes}

Outcome measures for efficacy were assessed 6 months after randomization by trained physicians and/ or psychologists. The primary outcome variables were i) adherence to the six sessions and ii) changes of global health-related quality of life (HRQoL) between randomization and the 6-month follow-up. Successful adherence was defined as participating in at least five distinct sessions within the 6 months after randomization. HRQoL was measured by the global score of the DISABKIDS-37 questionnaire (chronic generic module [19]). Secondary outcomes included changes from randomization to 6-month follow-up in different domains of HRQoL (on six subscales of the DISABKIDS questionnaire and the KIDSCREEN-52 questionnaire [20]), the depression score (Beck's Depression Inventory II (BDI-II) [21]), self-esteem (German version of the Rosenberg-Scale [22]), perceived stress (German version of the Perceived Stress Questionnaire (PSQ) with 20 items [23]), self-reported occupation (including school/university attendance, vocational training or work as a proxy for time spent outside the home), and self-reported physician/psychotherapist contacts.

Safety was measured by (serious) adverse events during the intervention. Participants were queried about adverse events at the end of each session and were offered to report them to the therapist individually. All events were documented and classified according to the Medical Dictionary for Regulatory Activities.

\section{Ethics}

The study protocol was approved by the human research ethics committees at the Universities of Ulm, Essen, Datteln, Berlin, and Leipzig. An independent Data Safety and Monitoring Board, consisting of experts in the fields of psychiatry, adolescent medicine and biometry/statistics, steadily supervised the process of recruitment and safety parameters according to the International Conference on Harmonisation of Technical Requirements for Registration of Pharmaceuticals for Human Use (Good Clinical Practice). Participants aged 18 and above gave written informed consent. In the case of minors, written informed consent was obtained independently from the adolescent and at least one caregiver.

\section{Sample Size}

Originally our study was designed to detect mean differences of 0.33 units of standardized effect sizes (Cohen's d) [17]. This resulted in $2 \times 175=350$ participants to be randomized to meet a power of $80 \%$, while allowing for a drop-out rate of $\sim 15 \%$. Due to substantially lower adherence rates, the trial was stopped early, including 119 participants only. Thus, the study was underpowered to detect the effect sizes of the original 
Mühlig et al.: A Structured, Manual-Based Low-Level Intervention vs. Treatment as Usual Evaluated in a Randomized Controlled Trial for Adolescents with Extreme Obesity - the STEREO Trial

plan. In fact, a study with $59+60=119$ participants has a power of $80 \%$ to detect mean differences $\geq 0.5$ units of standardized effect sizes for the HRQoL-related research question. Projected to the first of the two primary research questions (adherence rate), the power is $80 \%$ to detect differences between e.g. $10 \%$ and $33 \%$. The trial had no planned interim analyses.

\section{Randomization and Blinding}

Concealed allocation was performed by the independent Center for Clinical Trials Essen after participant registration; the validated software TENALEA (https://nl.tenalea.net/amc/ALEA/Login.aspx) was used for computer-based stratified (strata: center, gender, age ( $<18$ years, $\geq 18$ years), BMI class $\left(30-35 \mathrm{~kg} / \mathrm{m}^{2}\right.$, $\geq 35 \mathrm{~kg} / \mathrm{m}^{2}$ ) blocked randomization with randomly varying the block size. The study had a design without blinding.

\section{Statistical Methods}

The confirmatory test of the two hypotheses related to the primary outcomes followed a priori hierarchically ordered testing [24] to control the family-wise error rate in a strong sense at a significance level $\alpha$ (two-sided) of 5\%. As confirmatory test, we used Fishers' exact test for the first primary dichotomized outcome in successful ( $\geq$ five out of six sessions) and unsuccessful ( $<$ five sessions) adherence. For the second primary outcome, changes in the DISABKIDS global score between baseline and follow-up were analyzed. For all secondary outcomes we had to use methodologically simpler analyses (Welch's t tests and non-parametric Wilcoxon-Mann-Whitney test tests as sensitivity analysis (data not shown due to results highly similar to those obtained with Welch's t tests)) as compared to our initial plan [17] to address the limited number of observations. For the same reasons, we decided not to work with the change scores, but instead referred to the cross-sectional results at 6 months after randomization. The confirmatory tests were run in the intentionto-treat population. As sensitivity exploratory analyses, we re-ran the analyses in the per-protocol population (data not shown as results were identical due to only one protocol violation) and decided against stratified analyses due to the small sample size of participants with 6-month follow-up data. We report estimated effects, corresponding 95\% confidence intervals and two-sided p values. We applied no multiplicity adjustment for the exploratory sensitivity and secondary outcome analyses. All analyses were performed in R 3.1.1 or SAS 9.3.

\section{Results}

\section{Sample Characteristics}

Trial flow and baseline sample descriptions are shown in figure 1 and table 1. 30.0\% of the screened individuals fulfilled the inclusion criteria and were willing to participate in the RCT. Of 119 randomized participants (mean age $17.6 \pm 3.1$ years, $49.6 \%$ female), between 19 and 31 (19-26\% depending on the outcome) case report forms were available for the 6-month follow-up assessment. Mean baseline BMI of the whole study group was 40.4 \pm 7.0 $\mathrm{kg} / \mathrm{m}^{2}$.

\section{Efficacy}

Table 2 presents the results of the group comparisons for the primary and secondary outcomes. Depending on the scale of the outcome, either absolute risk reductions or mean differences are reported as estimated effect such that higher values of the differences indicate better performance of the manual-based low-level intervention. 
Mühlig et al.: A Structured, Manual-Based Low-Level Intervention vs. Treatment as Usual Evaluated in a Randomized Controlled Trial for Adolescents with Extreme Obesity - the STEREO Trial

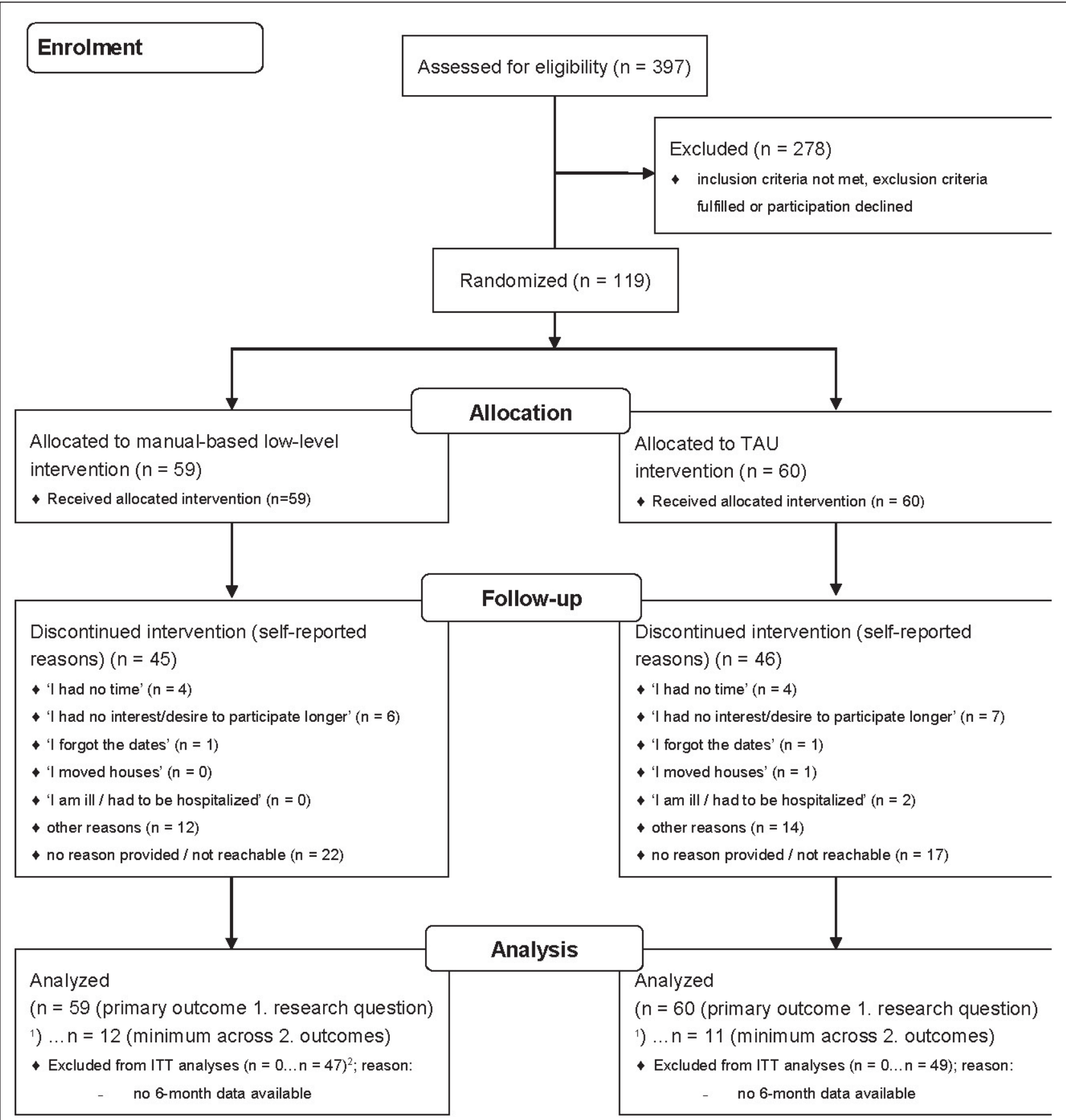

Fig. 1. CONSORT 2010 Flow Diagram [28] for the STEREO trial. ${ }^{1}$ Note that adherence as part of the primary outcome will be available for all allocated participants. ${ }^{2}$ One participant had to be excluded from the per protocol analyses due to violations of inclusion/exclusion criteria that were noted after the randomization.

Primary Outcomes

In each treatment group, only 14 participants (manual-based low-level intervention: $23.7 \%$ vs. TAU: $23.3 \%$ ) attended at least five of the six sessions. Thus, adherence did not differ between the treatment conditions in the confirmatory analysis (absolute risk 0.4\%; 95\% CI 
Mühlig et al.: A Structured, Manual-Based Low-Level Intervention vs. Treatment as Usual Evaluated in a Randomized Controlled Trial for Adolescents with Extreme Obesity - the STEREO Trial

Table 1. Baseline sample descriptions in the two intervention groups; either means \pm standard deviation or $\mathrm{n}(\%)$ are displayed

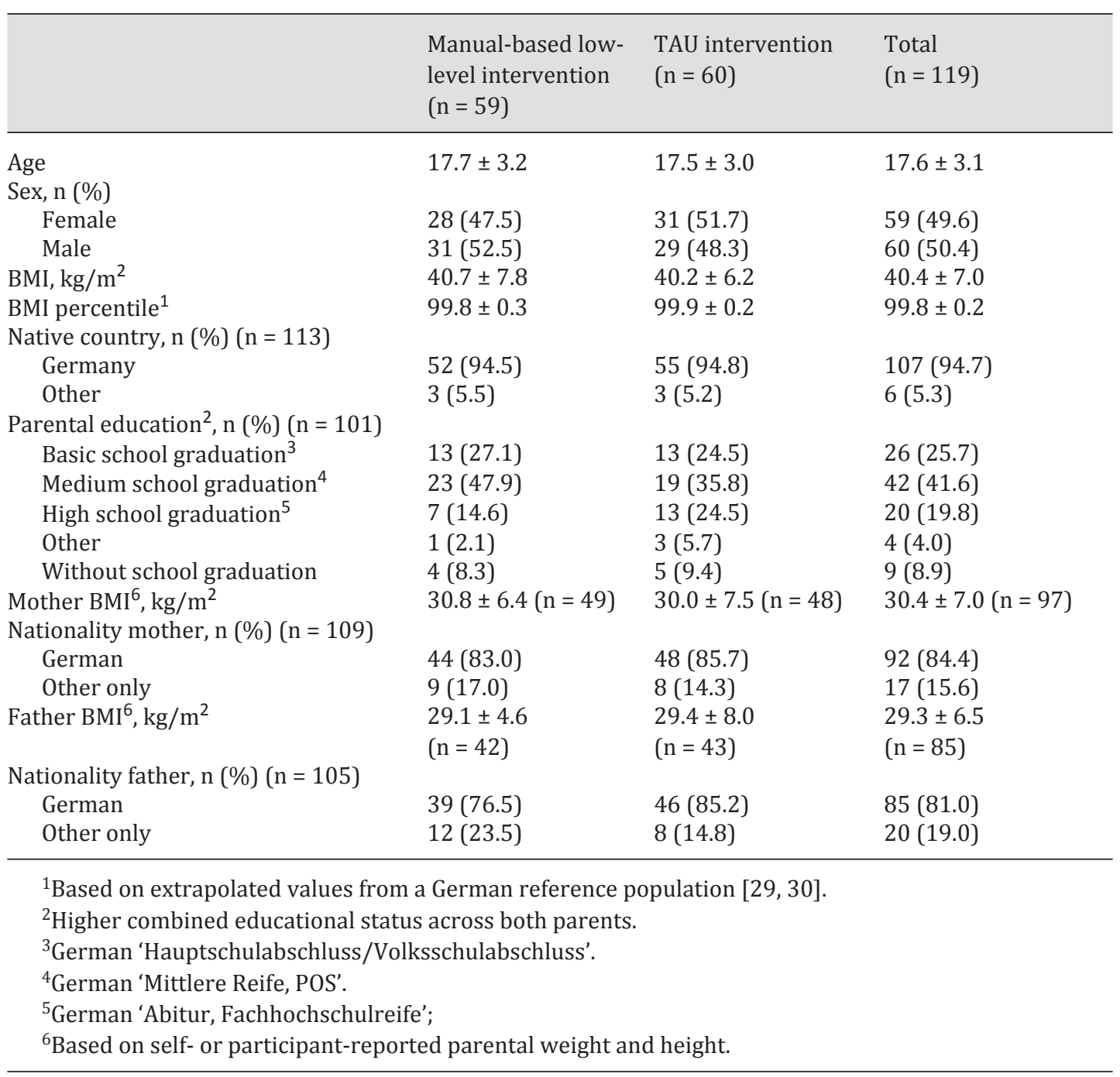

$-14.7 \%$ to $15.5 \% ; p=1.0$ ). Self-reported reasons for low adherence are listed in figure 1 . Similarly, we observed no evidence for a group difference in the global score of the DISABKIDS questionnaire as the second primary outcome measure (between group score difference at follow-up 8.1; 95\% CI -2.1 to $18.3 ; \mathrm{p}=0.11$ ).

\section{Secondary Outcomes}

We observed exploratory evidence for a group difference for the DISABKIDS subscale 'social exclusion', indicating a better performance of the manual-based low-level intervention group (score difference 15.5; 95\% CI 1.6-29.4; $\mathrm{p}=0.03$ ). For all other secondary outcomes, we found no evidence for group differences (all $p>0.05$ ) even though eight of the nine analyzed secondary outcomes ( $\mathrm{p}_{\text {binomial sign test }}=0.004$ ) indicated a descriptively better outcome in favor of the manual-based low-level intervention (table 2). 
Mühlig et al.: A Structured, Manual-Based Low-Level Intervention vs. Treatment as Usual Evaluated in a Randomized Controlled Trial for Adolescents with Extreme Obesity - the STEREO Trial

Table 2. Results of the primary and secondary outcomes by intervention group determined 6 months after randomization; either means \pm standard deviation or $\mathrm{n}(\%)$ are displayed

$\begin{array}{llll}\begin{array}{l}\text { Manual-based } \\ \text { low-level } \\ \text { intervention } \\ (\mathrm{n}=59)\end{array} & \begin{array}{l}\text { TAU intervention } \\ (\mathrm{n}=60)\end{array} & \begin{array}{l}\text { Estimated effect }{ }^{1} \\ (95 \% \mathrm{CI})\end{array} & \begin{array}{l}\text { p value } \\ \text { (two-sided) }\end{array} \\ \end{array}$

\section{Primary outcome(s)}

Adherence

Successful

Unsuccessful

HRQoL: DISABKIDS score (DCGM-37) ${ }^{2}$

$\begin{array}{llll}14(23.7) & 14(23.3) & 0.4 \%(-14.7 \% ; 15.5 \%) & 1.00 \\ 45(76.3) & 46(76.7) & 8.1(-2.1 ; 18.3) & 0.11 \\ 67.6 \pm 10.2(\mathrm{n}=12) & 59.5 \pm 13.6(\mathrm{n}=12) & 8.1\end{array}$

Secondary outcome(s)

HRQoL

DISABKIDS score ('independence') ${ }^{2}$

$67.6 \pm 19.7(\mathrm{n}=13) \quad 62.2 \pm 14.3(\mathrm{n}=13) \quad 5.4(-8.6 ; 19.4) \quad 0.43$

DISABKIDS score ('physical limitation') ${ }^{2}$

DISABKIDS score ('emotion') ${ }^{2}$

$69.6 \pm 17.0(n=13) \quad 61.5 \pm 9.5(n=13) \quad 8.0(-3.3 ; 19.4)$

$63.1 \pm 17.6(\mathrm{n}=13) \quad 49.2 \pm 21.8(\mathrm{n}=13) \quad 13.9(-2.2 ; 30.0)$

0.16

DISABKIDS score ('social exclusion') ${ }^{2}$

$79.3 \pm 12.7(\mathrm{n}=12) \quad 63.8 \pm 19.1(\mathrm{n}=12) \quad 15.5(1.6 ; 29.4)$

0.08

DISABKIDS score ('social inclusion') ${ }^{2}$

$63.2 \pm 12.1(\mathrm{n}=13) \quad 57.7 \pm 18.9(\mathrm{n}=13) \quad 5.5(-7.4 ; 18.4)$

0.03

KIDSCREEN-52 score $^{3}$

$67.8 \pm 13.2(n=12) \quad 61.3 \pm 16.4(n=11) \quad 6.5(-6.6 ; 19.6)$

0.39

Depression

BDI-II

Self-esteem

Rosenberg ${ }^{2}$

Perceived stress

PSQ overall score ${ }^{4}$

Self-reported occupation ${ }^{5}(n=16+14)$

School attendance

$7.3 \pm 5.4(\mathrm{n}=13) \quad 7.6 \pm 9.1(\mathrm{n}=13) \quad 0.3(-5.8 ; 6.5)$

0.31

Vocational training/studies

$67.8 \pm 16.1(\mathrm{n}=12) \quad 67.9 \pm 16.7(\mathrm{n}=14) \quad-0.1(-13.4 ; 13.2)$

0.92

Gap year to do voluntary work in the

social sector

Employed

Unemployed

$37.8 \pm 14.8(n=12)$

$40.7 \pm 17.2(n=14) 2.9(-10.0 ; 15.9)$

1.00

$8(50.0)$

8 (57.1)

2 (12.5)

$2(14.3)$

$1(6.3)$

$0(0.0)$

$0(0.0)$

$1(7.1)$

5 (31.3)

3 (21.4)

Self-reported physician / psychotherapist contacts $^{6}$

Physician $^{7}(\mathrm{n}=16+12)$

Psychotherapist $^{8}(\mathrm{n}=17+14)$

Hospital $^{9}$ ( $\left.n=16+14\right)$

$\begin{array}{ll}16(100.0) & 11(91.7) \\ 2(11.7) & 1(7.1) \\ 2(12.5) & 1(7.1)\end{array}$

ND

${ }^{1}$ Depending on the scale of the outcome either absolute risk reductions or mean differences are reported such that higher values of the differences indicate better performance of the experimental intervention.

${ }^{2}$ Transformed to a $0 ; 100$ scale (larger values indicate better outcomes).

${ }^{3}$ We created a global score which we transformed to a 0; 100 scale (larger values indicate better outcomes).

${ }^{4}$ Transformed to a 0; 100 scale (larger values indicate more perceived stress - i.e. a worse outcome).

${ }^{5}$ Current occupation 6 months after randomization.

${ }^{6}$ Multiple (yes/no) answers per participant were allowed.

${ }^{7}$ 'Did you contact a physician during the past 6 months?'

${ }^{8}$ 'Did you contact a physician during the last 6 months?'

9'Did you have inpatient treatment in the hospital during the past 6 months?' 
Mühlig et al.: A Structured, Manual-Based Low-Level Intervention vs. Treatment as Usual Evaluated in a Randomized Controlled Trial for Adolescents with Extreme Obesity - the STEREO Trial

Safety

We observed no serious adverse events during the trial period. 26 participants reported a total of 37 adverse events (manual-based low-level intervention: 19 events (nasopharyngitis and insomnia most frequently $(3 \times)$ reported); TAU: 18 events (nasopharyngitis and headache most frequently $(3 \times)$ reported). None was judged as likely related to the intervention.

\section{Discussion}

This trial investigated the adherence to six sessions, efficacy and safety of a manual-based low-level intervention aimed at coping with obesity and its acceptance compared to TAU (weight loss treatment). Only a small proportion of adolescents and young adults participated in our program. Adherence was low and did not differ between the treatment groups. The participation rates were comparable to those in other RCTs. Recent systematic reviews [2, 3] reported 'drop-out' rates of up to $42 \%$ or $50 \%$, and 'losses to follow-up' of up to $43 \%$ or $71 \%$. The adherence of adolescents and young adults compared to children could be even lower because of less parental support. Similarly, prior trials have revealed a low treatment interest in families of obese children [25].

Center-specific subanalyses revealed that the unemployed adolescents and young adults recruited in the job center exhibited a relatively higher adherence than those recruited from the pediatric university hospitals (32.2\% vs. $20.5 \%)$. Possible explanations might be that these unemployed subjects were substantially older ( $21.6 \pm 2.3$ vs. $16.2 \pm 1.8$ years) and could have been more motivated because of their difficult psychosocial situation. Another possible explanation may be the lower treatment barrier, as the intervention was offered at a job center, rather than in a medical institution. Contrastingly, in another study a small proportion of families with low socioeconomic status was enrolled into obesity treatment programs [26]. Indeed, the reduction of treatment barriers for individuals with low socioeconomic status forms an important issue for health care policies [27].

For the second primary and the secondary outcomes, we also observed no evidence for a treatment effect. The secondary primary outcome, the HRQoL global score measured by the DISABKIDS questionnaire, was slightly higher in the manual-based low-level intervention group but did not significantly differ between the treatment groups. For the DISABKIDS subscale 'social exclusion', we observed explorative evidence for a better performance of the manual-based low-level intervention. If not due to multiple testing, this result may be attributed to a reduction of social isolation achieved by teaching social competence in the manual-based low-level intervention. Besides that, a tendency for a better outcome of the manual-based low-level intervention was observed in most of the secondary psychosocial outcome measures based on the respective mean values. On the other hand, positive group interactions and thus reduction of social isolation could have led to a similar participation rate and improvement of psychosocial functioning in the TAU group. In a comparable trial [13], both treatment groups (social skills training + TAU vs. motivational interviewing + TAU) improved their self-efficacy after 6 months.

\section{Strengths and Limitations}

To our knowledge, this is the first RCT assessing adherence defined as the participation rate as a primary outcome measure in an intervention not aiming at weight loss. With our suggested multi-leveled program, consisting of the manual-based low-level intervention and 
Mühlig et al.: A Structured, Manual-Based Low-Level Intervention vs. Treatment as Usual Evaluated in a Randomized Controlled Trial for Adolescents with Extreme Obesity - the STEREO Trial

a subsequent information and preparation program on bariatric surgery [17], we propose a novel treatment approach which might help identifying adolescents and young adults eligible for bariatric surgery. Only individuals exhibiting a solid pre-surgical adherence are presumed to be willing/able to undergo continuous post-surgical monitoring which may help to prevent long-term risks and complications of bariatric surgery, especially in adolescents and young adults. Besides this, helping this age group to cope with psychosocial impairments forms an important task as extreme obesity is a chronic condition. Further strengths of this study encompass the multi-centered randomized study design, the use of a TAU arm consistent with clinical guidelines of obesity treatment [18], the involvement of multiple recruitment pathways (including facilitation of treatment access for individuals with low socioeconomic status) and the involvement of a multidisciplinary team and a Data Safety and Monitoring Board with profound clinical trial experience.

The most important limitation was the small sample size leading to a substantial loss of statistical power. Despite the use of multiple recruitment pathways and initiatives to boost recruitment we did not reach the proposed sample size. Consequently, the application of statistical methods was limited. To allow for exploratory analyses of the secondary outcomes, we did not adjust them for multiple testing. Due to the small sample size and heterogeneity of the study centers (e.g. recruitment pathways, age), results cannot be generalized to adolescents/young adults with extreme obesity. A relatively high proportion of participants discontinued the intervention, and, even though this was addressed by our primary analysis strategy, it is still possible that an attrition bias impacted the questionnaire-related outcomes. A potential performance bias was reduced by a standardized manual-based approach carried out at all study centers. Due to a study without blinding, a detection bias cannot be ruled out.

\section{Conclusion}

Overall, this study revealed no evidence for treatment effects in adherence or HRQoL when comparing a manual-based psychological intervention against TAU for adolescents and young adults with extreme obesity. On one hand, the participation in a psychological intervention may help to overcome social isolation and cope with impairments in social life, but further confirmatory trials are needed to substantiate this explorative observation. On the other hand, the major problem of a low adherence rate in treatment programs for adolescents with (extreme) obesity [2,3] could not be solved by offering a coping-oriented psychological treatment approach in our study. The low adherence rate highlights the importance of assessing adherence in future trials and clinical practice, especially for socioeconomically disadvantaged and those adolescents and young adults presenting for bariatric surgery. The subjective needs of adolescents and young adults with (extreme) obesity to feel supported and not stigmatized should form a major focus.

\section{Funding}

This study was funded by the German Federal Ministry of Education and Research (BMBF; project funding reference number: 01GI1120A/B) as part of the German Competence Network Obesity (Consortium 'Youth with Extreme Obesity'). AS was also supported by a BMBF grant from the Center for Sepsis Control and Care (FKZ: 01E01002; 01E01502). 
Mühlig et al.: A Structured, Manual-Based Low-Level Intervention vs. Treatment as Usual Evaluated in a Randomized Controlled Trial for Adolescents with Extreme Obesity - the STEREO Trial

\section{Ethics Approval and Consent to Participate}

The study protocol was approved by the human research ethics committees at the Universities of Ulm, Essen, Datteln, Berlin, and Leipzig. The STEREO trial is registered at 'clinicaltrials.gov' (identification number: NCT01703273; Registered 7 August, 2012). Parts of the trial protocol have been published [17].

\section{Acknowledgments}

We thank all participants of this study and we also thank Kerstin Konrad, Justine Rochon, and Detlef Kunze for their contribution to this study as members of the Data Monitoring and Safety Board.

\section{Disclosure Statement}

YM, UG, RH, BL, DL, AM, CO, TR, CT, MW, and JH report grants from the German Federal Ministry of Education and Research (BMBF) received by their institution during the conduction of the study (project funding no. 01GI1120A/B). AS reports grants from the German Federal Ministry of Education and Research (BMBF; project funding no. 01GI1120A/B) and from the Center of Sepsis Control and Care (CSCC; project funding no. 01E01002, 01E01502) received by his institution during the conduction of the study. AB, RWH, WK, MN, SW, and BW have nothing to disclose.

\section{References}

1 Ng M, Fleming T, Robinson M, : Global, regional, and national prevalence of overweight and obesity in children and adults during 1980-2013:a systematic analysis for the Global Burden of Disease Study 2013. Lancet 2014; 384:766-781.

2 Oude Luttikhuis H, Baur L, Jansen H, Shrewsbury VA, O'Malley C, Stolk RP, Summerbell CD: Interventions for treating obesity in children (review). Cochrane Database of Syst Rev 2009;3:1-57.

3 Mühlig Y, Wabitsch M, Moss A, Hebebrand J: Weight loss in children and adolescents - a systematic review and evaluation of conservative, non-pharmacological obesity treatment programs. Dtsch Arztebl Int 2014;111: 818-824.

4 Knop C, Singer V, Uysal Y, Schaefer A, Wolters B, Reinehr T: Extremely obese children respond better than extremely obese adolescents to lifestyle interventions. Pediatr Obes 2013;10:7-14.

5 Han JC, Lawlor DA, Kimm SYS: Childhood obesity - 2010: progress and challenges. Lancet 2010;375:17371748.

6 Hebebrand J, Herpertz-Dahlmann B: Psychological and psychiatric aspects of pediatric obesity. Child Adolesc Psychiatr Clin N Am 2009;18:49-65.

7 Puhl RM, Latner JD: Stigma, obesity, and the health of the nation's children. Psychol Bull 2007;133:557-580.

8 Puhl RM, Heuer CA: Weight bias: a review and update. Obesity (Silver Spring) 2009;17:941-964.

9 Giel KE, Thiel A, Teufel M, Mayer J, Zipfel S: Weight bias in work settings - a qualitative review. Obes Facts 2010;3:33-40.

10 Wasserman $\mathrm{H}$, Inge TH: Bariatric surgery in obese adolescents: opportunities and challenges. Pediatr Ann 2014;43:e230-e236.

11 Papadia FS, Adami GF, Marinari GM, Camerini G, Scopinaro N: Bariatric surgery in adolescents: a long-term follow-up study. Surg Obes Relat Dis 2007;3:465-468.

12 Lennerz BS, Wabitsch M, Lippert H, Wolff S, Knoll C, Weiner R, Manger T, Kiess W, Stroh C: Bariatric surgery in adolescents and young adults-safety and effectiveness in a cohort of 345 patients. Int J Obes (Lond) 2013; 182:1-7.

13 Walpole B, Dettmer E, Morrongiello BA, McCrindle BW, Hamilton J: Motivational interviewing to enhance selfefficacy and promote weight loss in overweight and obese adolescents: a randomized controlled trial. J Pediatr Psychol 2013;38:944-953.

14 Bean MK, Powell P, Quinoy A, Ingersoll K, Wickham EP 3rd, Mazzeo SE: Motivational interviewing targeting diet and physical activity improves adherence to paediatric obesity treatment: results from the MI Values randomized controlled trial. Pediatr Obes 2014;10:118-125.

15 Theim KR, Sinton MM, Goldschmidt AB, van Buren DJ, Doyle AC, Saelens BE, Stein RI, Epstein LH, Wilfley DE: Adherence to behavioral targets and treatment attendance during a pediatric weight control trial. Obesity (Silver Spring) 2013;21:394-397. 
Mühlig et al.: A Structured, Manual-Based Low-Level Intervention vs. Treatment as Usual Evaluated in a Randomized Controlled Trial for Adolescents with Extreme Obesity - the STEREO Trial

16 De Niet J, Timman R, Bauer S, van den Akker E, Buijks H, de Klerk C, Kordy H, Passchier J: The effect of a short message service maintenance treatment on body mass index and psychological well-being in overweight and obese children: a randomized controlled trial. Pediatr Obes 2012;7:205-219.

17 Wabitsch M, Moss A, Reinehr T, Wiegand S, Kiess W, Scherag A, Holl R, Holle R, Hebebrand J: Medical and psychosocial implications of adolescent extreme obesity - acceptance and effects of structured care, short: Youth with Extreme Obesity Study (YES). BMC Pub Health 2013;13:789.

18 Wabitsch M, Moss A (federführend für die Arbeitsgemeinschaft Adipositas im Kindes- und Jugendalter): Evidenzbasierte Leitlinie zur Therapie der Adipositas im Kindes- und Jugendalter. S3-Leitlinie, Version 2009. www.aga.adipositas-gesellschaft.de/fileadmin/PDF/Leitlinien/Leitlinie-AGA-S3-2009.pdf (last accessed July 31, 2017).

19 Schmidt S, Petersen C, Mühlan H, Simeoni, MC, Debensason D, Thyen U, Müller-Godeffroy E, Vidalis A, Tsanakas J, Hatziagorou E, Karagianni P, Koopmann H, Baars R, Chaplin J, Power M, Atherton C, Hoare P, Quittan M, Schuhfried 0, Bullinger M: The DISABKIDS Questionnaires. Quality of life questionnaires for children with chronic conditions. Handbook. Lengerich, Pabst Science Publishers, 2006.

20 Ravens-Sieberer U, Gosch A, Rajmil L: KIDSCREEN-52 quality-of-life measure for children and adolescents. Expert Rev Pharmacoecon Outcomes Res 2005;5:353-364.

21 Beck AT, Steer RA, Brown GK: Manual for the Beck Depression Inventory-II. San Antonio, Psychological Corporation, 1996.

22 Collani G, Herzberg PY: Eine revidierte Fassung der deutschsprachigen Skala zum Selbstwertgefühl von Rosenberg. Zeitschrift für Differentielle und Diagnostische Psychologie 2003;24:3-7.

23 Fliege H, Rose M, Arck P, Walter OB, Kocalevent RD, Klapp BF: The Perceived Stress Questionnaire (PSQ) reconsidered: validation and reference values from different clinical and healthy adult samples. Psychosom Med 2005;67:78-88.

24 Maurer W, Hothorn L, Lehmacher W: Multiple comparisons in drug clinical trials and preclinical assays: a priori ordered hypothesis; in Vollmar J (ed): Biometrie in der chemisch-pharmazeutischen Industrie, vol 6. Stuttgart, Fischer, 1995, pp. 3-18.

25 Finne E, Reinehr T, Schaefer A, Winkel K, Kolip P: Overweight children and adolescents - is there a subjective need for treatment? Int J Public Health 2009;54:112-116.

26 Röbl M, de Souza M, Schiel R, Gellhaus I, Zwiauer K, Holl RW, Wiegand S: The key role of psychosocial risk on therapeutic outcome in obese children and adolescents. Results from a longitudinal multicenter study. Obes Facts 2013;6:297-305.

27 Halloran K, Padwal RS, Johnson-Stoklossa C, Sharma AM, Birch DW: Income status and approval for bariatric surgery in a publicly funded regional obesity program. Obes Surg 2011;21:373-378.

28 Schulz KF, Altman D, Moher D, for the CONSORT Group: CONSORT 2010 Statement: updated guidelines for reporting parallel group randomised trials. BMC Med 2010;8:18.

29 Kromeyer-Hauschild K, Wabitsch M, Geller F, Ziegler A, Geiß HC, Hesse V, Hippel V, Jaeger U, Johnsen D, Kiess W, Korte W, Kunze D, Menner K, Müller M, Niemann-Pilatus, A, Remer T, Schaefer F, Wittchen HU, Zabransky S, Zellner K, Hebebrand J: Perzentile für den Body Mass Index für das Kindes- und Jugendalter unter Heranziehung verschiedener deutscher Stichproben. Monatsschr Kinderheilk 2001;149:807-818.

30 Max Rubner Institut, Bundesforschungsinstitut für Ernährung und Lebensmittel: Nationale Verzehrsstudie II Ergebnisbericht Teil 1, 2008. www.mri.bund.de/fileadmin/Institute/EV/NVS_II_Abschlussbericht_Teil_1_mit_ Ergaenzungsbericht.pdf (last accessed July 31, 2017). 